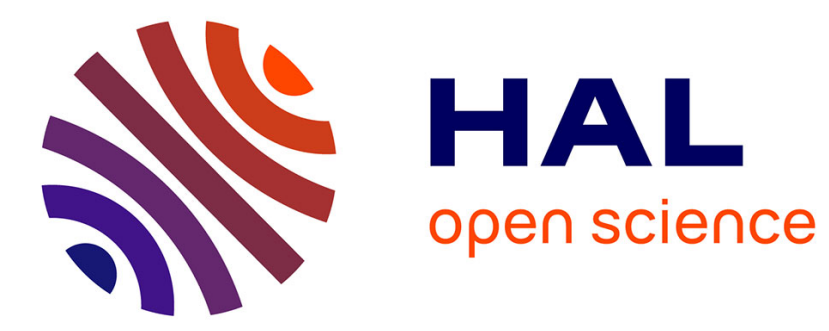

\title{
Strong Thermal and Electrostatic Manipulation of the Casimir Force in Graphene Multilayers
}

\author{
Chahine Abbas, Brahim Guizal, Mauro Antezza
}

\section{To cite this version:}

Chahine Abbas, Brahim Guizal, Mauro Antezza. Strong Thermal and Electrostatic Manipulation of the Casimir Force in Graphene Multilayers. Physical Review Letters, 2017, 118, pp.126101. 10.1103/PhysRevLett.118.126101 . hal-01494732

\section{HAL Id: hal-01494732 \\ https://hal.science/hal-01494732}

Submitted on 13 Oct 2020

HAL is a multi-disciplinary open access archive for the deposit and dissemination of scientific research documents, whether they are published or not. The documents may come from teaching and research institutions in France or abroad, or from public or private research centers.
L'archive ouverte pluridisciplinaire HAL, est destinée au dépôt et à la diffusion de documents scientifiques de niveau recherche, publiés ou non, émanant des établissements d'enseignement et de recherche français ou étrangers, des laboratoires publics ou privés. 


\title{
Strong Thermal and Electrostatic Manipulation of the Casimir Force in Graphene Multilayers
}

\author{
Chahine Abbas, ${ }^{1}$ Brahim Guizal, ${ }^{1}$ and Mauro Antezza, ${ }^{1,2, *}$ \\ ${ }^{1}$ Laboratoire Charles Coulomb (L2C), UMR 5221 CNRS-Université de Montpellier, F-34095 Montpellier, France \\ ${ }^{2}$ Institut Universitaire de France, 1 rue Descartes, F-75231 Paris, France
}

(Received 23 December 2016; published 23 March 2017)

\begin{abstract}
We show that graphene-dielectric multilayers give rise to an unusual tunability of the Casimir-Lifshitz forces and allow to easily realize completely different regimes within the same structure. Concerning thermal effects, graphene-dielectric multilayers take advantage of the anomalous features predicted for isolated suspended graphene sheets, even though they are considerably affected by the presence of the dielectric substrate. They can also achieve the anomalous nonmonotonic thermal metallic behavior by increasing the graphene sheets density and their Fermi level. In addition to a strong thermal modulation occurring at short separations, in a region where the force is orders of magnitude larger than the one occurring at large distances, the force can be also adjusted by varying the number of graphene layers as well as their Fermi levels, allowing for relevant force amplifications which can be tuned, very rapidly and in situ, by simply applying an electric potential. Our predictions can be relevant for both Casimir experiments and micro- or nanoelectromechanical systems and in new devices for technological applications.
\end{abstract}

DOI: 10.1103/PhysRevLett.118.126101

The Casimir-Lifshitz pressure (CLP) occurring between closely spaced bodies is a mechanical manifestation of both quantum vacuum and thermal fluctuations of radiation and matter fields [1-3]. It is the object of large theoretical and experimental interest [4] for both its fundamental and applicative implications. In particular, on the applicative side, such a force has a clear impact in micro- and nano (electro)mechanical systems (MEMS and NEMS, respectively), where it plays a dominant role at small separations [5]. For parallel planar structures separated by a distance $d$, the CLP can be expressed as [2]

$$
\begin{aligned}
P= & -\frac{\hbar}{2 \pi^{2}} \int_{0}^{\infty} d \omega \operatorname{coth}\left(\frac{\hbar \omega}{2 k_{B} T}\right) \\
& \times \operatorname{Re}\left[\int_{0}^{\infty} d Q Q q_{z} \sum_{p}\left(\frac{e^{-2 i q_{z} d}}{R_{p}^{(1)} R_{p}^{(2)}}-1\right)^{-1}\right],
\end{aligned}
$$

where $p=$ TE, TM stands for the two light polarizations (transverse electric and transverse magnetic) and $q_{z}=$ $\sqrt{(\omega / c)^{2}-Q^{2}}$ and $R_{p}^{(i)}(Q, \omega, T)$ are the $z$ component of the vacuum wave vector and the reflection coefficient of bodies $i=1,2$, respectively. The integral is over the parallel-plane wave vector component $Q$.

Equation (1) shows that the CLP can be tuned by modifying the bodies' reflection coefficients or by varying the temperature $T$. In practice, thermal manipulation has been always considered as noneffective: At short separations $(d \leq 1 \mu \mathrm{m})$, where the CLP is stronger, thermal effects are very small compared to vacuum $(T=0 \mathrm{~K})$ ones. Remarkably, a thermal metal anomaly (TMA) has been predicted: For metals at intermediate separations

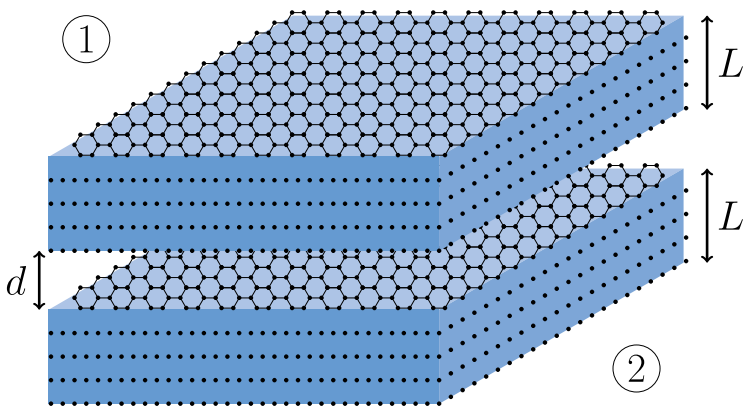

FIG. 1. Graphene-based multilayer scheme.

$(\simeq 1 \mu \mathrm{m})$, contrary to dielectrics, the CLP decreases with an increasing temperature [6]. Thermal effects dominate only at very large separations $d \gg \lambda_{T}=\hbar c /\left(k_{B} T\right)(\approx 7 \mu \mathrm{m}$ at room temperature), where the force reduces to the Lifshitz limit [for metals $P_{\text {Lif }}=-k_{B} T \zeta(3) /\left(8 \pi d^{3}\right)$ [3]] and is already extremely weak and very hard to measure [7-11]. For this reason, almost all research efforts focused on changing the reflection coefficients by using more complex geometries (recently, large interest has been devoted to gratings [12-18]) and materials (like topological insulators [19], metamaterials [20], switchable mirrors [21], and others [22]).

Recently, the availability of graphene, with its peculiar transport and optical properties [23], stimulated both theoretical [24-30] and experimental [31] investigations of the CLP involving graphene sheets, with applications in nanophotonics and optomechanical systems [32].

Remarkably, the CLP between two suspended parallel graphene sheets has been predicted [25] to reach the 
Lifshitz metallic behavior $P_{\text {Lif }}$ at very small separations $d \gg \chi_{T}=\hbar v_{F} /\left(k_{B} T\right) \ll \lambda_{T}$, since the Fermi velocity is much smaller than $c\left(v_{F} \simeq c / 300\right)$. A natural question, then, is to what extent this striking thermal graphene anomaly (TGA) persists in typical realistic Casimir experimental conditions, which require the presence of substrates $[11,31]$ in a mixed graphene-dielectric configuration. This issue is also crucial for technological applications in MEMS and NEMS and in microoptomechanical devices, calling for a specific investigation due to the nonadditive nature of the CLP.

In this Letter, by simply introducing a dielectric substrate (we consider a general parallel-plane graphene-dielectric multilayer configuration), we propose a setting which allows several important modulations of the CLP and opens to genuine technological applications. Furthermore, it allows compatibility with existing Casimir experiments and naturally guarantees the flatness and parallelism assumed in the model [25].

First, we show that the TGA strongly deviates from the ideal suspended-graphene configuration, still remaining large enough to thermally modulate the force at separations $\sim 200 \mathrm{~nm}$, where the CLP is strong and typically measured. Second, we show that, by increasing the density of the graphene layers in the dielectric host, we recover the TMA once the graphene is doped. Finally, we show that the same system allows an easy, strong, and rapid CLP electrostatic tunability in situ by modulating the graphene conductivity with an applied voltage to the graphene sheets.

All these effects are particularly relevant for experiments, since they allow one, contrary to almost all known configurations, to dynamically change the force in the same experimental device without changing the geometry or materials.

Physical system and model.-We consider the interaction between two identical parallel graphene multilayers imbedded in two dielectric slabs separated by a distance $d$ (see Fig. 1). Each slab has a thickness $L$ and is loaded with $N_{g}$ equally spaced graphene sheets dividing the slab into $N=N_{g}-1$ layers. The dielectric layers are characterized by their permittivity $\varepsilon(\omega)$, and the graphene sheets by their conductivity $\sigma\left(\omega, T, \mu_{F}\right)$ (where $\mu_{F}$ is the Fermi level).

While Eq. (1) is useful for understanding the roles of the different parameters, for computational efficiency we would rather use its frequency complex-rotated version $\left(\omega=i \xi_{n}\right)[2]$

$$
P=-\frac{k_{B} T}{\pi} \sum_{n=0}^{\infty} \int_{0}^{\infty} d Q Q q \sum_{p}\left[\frac{e^{2 q d}}{R_{p}^{(1)} R_{p}^{(2)}}-1\right]^{-1},
$$

where the prime on the sum means that the $n=0$ term is divided by $2, \xi_{n}=2 \pi n k_{B} T / \hbar$ are the Matsubara frequencies, $q=\sqrt{\left(\xi_{n} / c\right)^{2}+Q^{2}}$, and $R_{p}^{(i)}\left(Q, i \xi_{n}, T\right)$ are the frequency-rotated reflection coefficients. In order to compute the graphene multilayer reflection coefficients, we implemented the scattering matrix algorithm (see [33] for details) because of its outstanding stability with respect to all the parameters of the problem.

In the following, we will consider $\mathrm{SiO}_{2}$ slabs with permittivity $\varepsilon(\omega)=\varepsilon_{R}(\omega)+i \varepsilon_{I}(\omega)$ taken from Ref. [34], which at the Matsubara frequencies becomes $\varepsilon\left(i \xi_{n}\right)=1+$ $(2 / \pi) \int_{0}^{\infty}\left[\omega \varepsilon_{I}(\omega) / \omega^{2}+\xi_{n}^{2}\right] d \omega$ [35]. The graphene sheets conductivity $\sigma(\omega)=\sigma_{R}(\omega)+i \sigma_{I}(\omega)$ is the sum of the intraband and interband contributions [36] (see also [37-39]) and at Matsubara frequencies takes the form [40]

$$
\begin{aligned}
\sigma\left(i \xi_{n}\right) & =\sigma_{\text {intra }}\left(i \xi_{n}\right)+\sigma_{\text {inter }}\left(i \xi_{n}\right), \\
\sigma_{\text {intra }}\left(i \xi_{n}\right) & =\frac{8 \sigma_{0} k_{B} T}{\pi\left(\hbar \xi_{n}+\hbar \Gamma\right)} \ln \left[2 \cosh \left(\frac{\mu_{F}}{2 k_{B} T}\right)\right], \\
\sigma_{\text {inter }}\left(i \xi_{n}\right) & =\frac{\sigma_{0} 4 \hbar \xi_{n}}{\pi} \int_{0}^{\infty} \frac{\mathcal{G}(x)}{\left(\hbar \xi_{n}\right)^{2}+4 x^{2}} d x .
\end{aligned}
$$

Here, $\sigma_{0}=e^{2} /(4 \hbar), e$ is the electron charge, the Fermi level $\mu_{F}$ (typically between 0 and $1 \mathrm{eV}$ ) can be modulated by applying a bias voltage or by chemical doping, $\mathcal{G}(x)=f(-x)-f(x)=\sinh \left(x / k_{B} T\right) /\left[\cosh \left(\mu_{F} / k_{B} T\right)+\right.$ $\left.\cosh \left(x / k_{B} T\right)\right]$ with $f(x)=\left\{\exp \left[\left(x-\mu_{F}\right) /\left(k_{B} T\right)\right]+1\right\}^{-1}$, and $\Gamma$ accounts for relaxation mechanisms (we use $\Gamma=10^{13} \mathrm{rad} / \mathrm{s}$ ).

Thermal and electrostatic modulation.-We first focus on the influence of the temperature variation for graphene multilayer structures in Fig. 2, where we evaluate, as a function of the separation distance, the relative variation of the CLP for two different temperatures, namely, $T=200 \mathrm{~K}$ and $T=400 \mathrm{~K}$, with respect to the pressure at $T=300 \mathrm{~K}$ used as a reference. In Fig. 2(a), we compare the CLP between two dielectric $\mathrm{SiO}_{2}$ slabs, two parallel suspended graphene sheets, and two identical graphenedielectric multilayers with $N=1$ (hence, with $N_{g}=2$ graphene sheets each) and with $N=10$.

We see that the CLP in graphene-dielectric multilayers strongly deviates from that in the suspended parallel graphene-graphene configuration, whose almost constant behavior in Fig. 2(a) reflects the rapid TGA saturation of the CLP to the Lifshitz limit [25]. It is worth noticing that, in the case of slabs, to reach a $10 \%$ relative variation, very large separations are required $(d \simeq 2 \mu \mathrm{m}$ for both $T=$ $200 \mathrm{~K}$ and $T=400 \mathrm{~K}$ ), where the total CLP is already negligible, making elusive the measurement of thermal effects. This appears clearly in Fig. 3, where at $d=2 \mu \mathrm{m}$ the slab-slab CLP is $\simeq-10^{-8} \mathrm{nN} / \mu \mathrm{m}^{2}$. Remarkably, for graphene-dielectric multilayers (for both $N=1$ and $N=10$ ), a $10 \%$ relative variation is already reached at $d \simeq 200 \mathrm{~nm}$. At this distance (which is typical in Casimir experiments), the CLP for graphene multilayers is $\simeq-10^{-4} \mathrm{nN} / \mu \mathrm{m}^{2}$ (see Fig. 3), which is 4 orders of magnitude larger than for the simple slab configurations. This precisely opens to the possibility of measuring thermal 

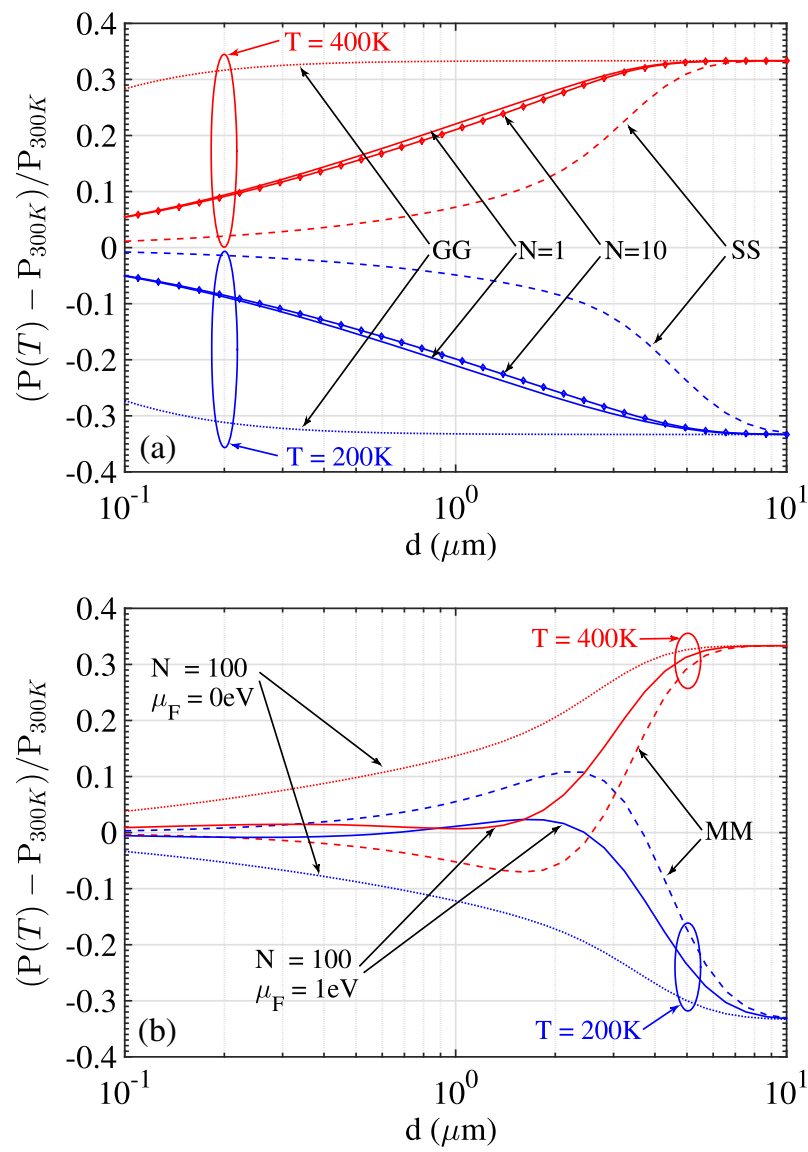

FIG. 2. Relative variation of $P$ when $T$ varies $[T=200 \mathrm{~K}$ (blue lines) and $T=400 \mathrm{~K}$ (red lines)]. The CLP at $T=300 \mathrm{~K}$ is taken as a reference, and $L=1 \mu \mathrm{m}$. (a) Slabs (SS, dashed curves), suspended graphene sheets (GG, dotted curves), and graphene-dielectric multilayers with $\mu_{F}=0 \mathrm{eV}(N=1$, solid curve; $N=10$, solid curve with diamonds). (b) Gold (MM, dashed curves) and graphene-dielectric multilayers with $N=100$ ( $\mu=0 \mathrm{eV}$, dotted curve; $\mu=1 \mathrm{eV}$, solid curve).

effects, especially at small distances, and to thermally manipulate the force within standard Casimir experimental setups.

In Fig. 2(b), we compare the CLP for two gold slabs [41] and two $N=100$ graphene-dielectric multilayers with $\mu=0 \mathrm{eV}$ and $\mu=1 \mathrm{eV}$. We clearly see that for $\mu=0 \mathrm{eV}$ the relative thermal variation for $N=100$ is weaker than for $N=1$ and $N=10$ [Fig. 2(a)], showing that by increasing $N$ the relative thermal effect decreases, while its absolute value increases [see Fig. 4(d)] and that both $N=1$ and $N=10$ are almost equally good candidates to measure the CLP relative thermal variations. For $\mu=$ $1 \mathrm{eV}$ it becomes nonmonotonic, acquiring the TMA behavior shown by gold. In that case, the collective behavior of the 2D embedded graphene sheets makes the graphene-dielectric multilayer structure equivalent to an effective 3D metal.

Let us now focus on other ways to tune the CLP which are offered by such structures. In the first line in Fig. 4, we show how a change in the Fermi level $\mu_{F}$, which can be done in situ

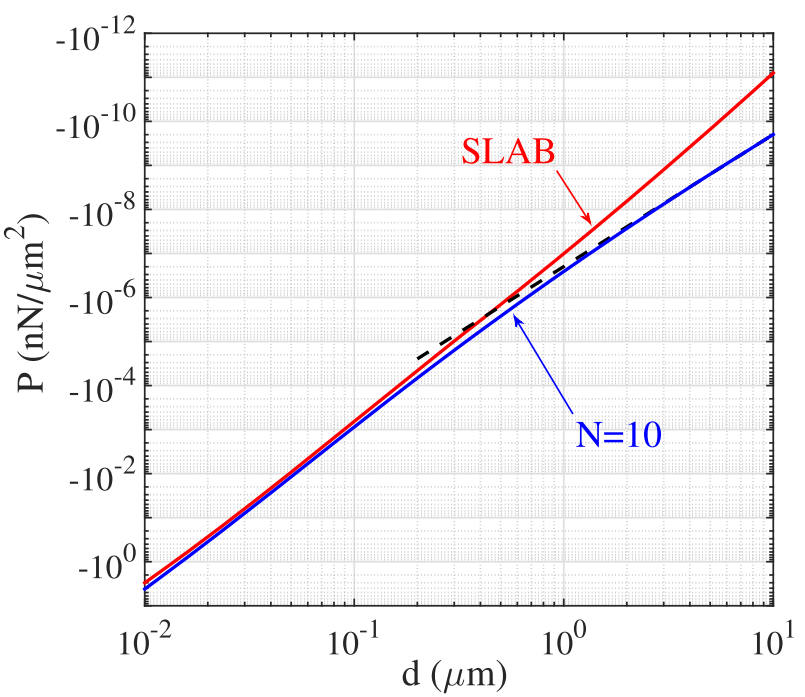

FIG. 3. CLP for $L=1 \mu \mathrm{m}$ and $T=300 \mathrm{~K}$, for different structures: $\mathrm{SiO}_{2}$ slabs (red solid line), graphene multilayers with $N=10$ and $\mu_{F}=0 \mathrm{eV}$ (blue solid line), and the Lifshitz limit for metals $P_{\text {Lif }}$ (black dashed line).

and dynamically, affects the CLP strength. We fix the number of layers $[N=10,50$, and 100 for Figs. 4(a), 4(b), and 4(c), respectively] and calculate, as a function of the distance, the relative variation of the CLP at increasing values of $\mu_{F}$, by normalizing with respect to the pressure at $\mu_{F}=0 \mathrm{eV}$.

We see that, already with $N=10$, the relative variation can reach $20 \%$ [Fig. 4(a)], and for $N=100$ a remarkable
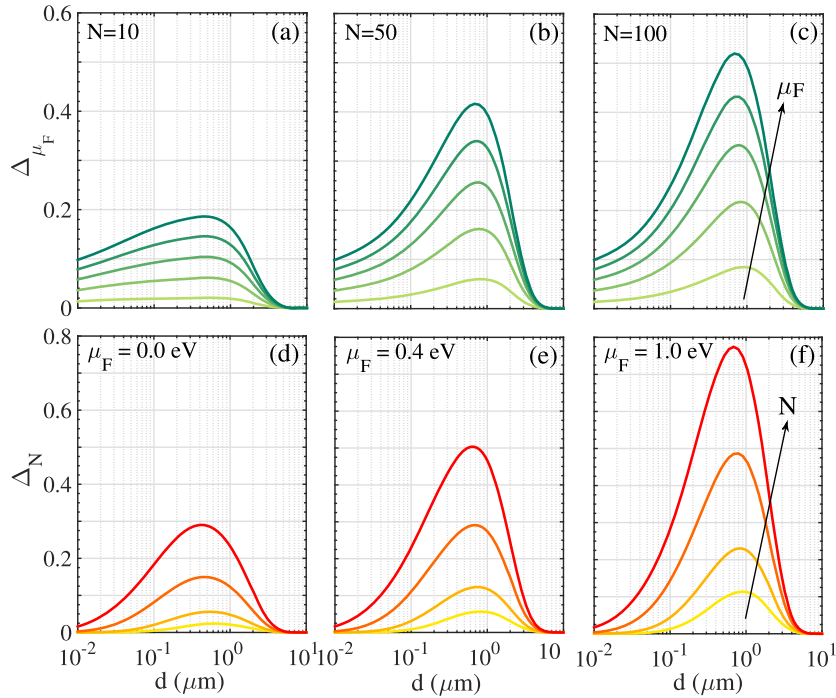

FIG. 4. Relative variation of the CLP as a function of the distance between the two multilayers, for $T=300 \mathrm{~K}$ and $L=1 \mu \mathrm{m}$. (a)-(c) We plot $\Delta_{\mu_{F}}=\mid\left[P\left(\mu_{F}\right)-P\left(\mu_{F}=0\right)\right] /$ $P\left(\mu_{F}=0\right) \mid$ for $\mu_{F}=0.2,0.4,0.6,0.8$, and $1 \mathrm{eV}$, with $N=10$ (a), $N=50$ (b), and $N=100$ (c). (d)-(f) We plot $\Delta_{N}=$ $|[P(N)-P(N=1)] / P(N=1)|$ for $N=10,20,50$, and 100, with $\mu_{F}=0 \mathrm{eV}(\mathrm{d}), \mu_{F}=0.4 \mathrm{eV}$ (e), and $\mu_{F}=1 \mathrm{eV}$ (f). 


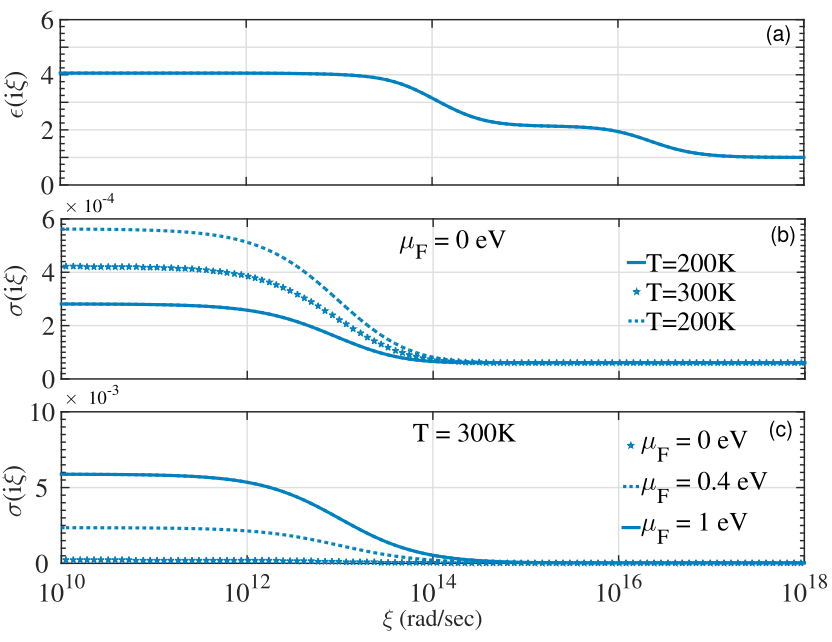

FIG. 5. $\mathrm{SiO}_{2}$ permittivity (a) and graphene conductivity (b),(c) at imaginary frequencies.

variation $>50 \%$ can be obtained by continuously tuning $\mu_{F}$ up to $1 \mathrm{eV}$ [Fig. 4(c)]. In the second line in Fig. 4, we show how much the CLP depends on $N$. We fix the Fermi level $\left[\mu_{F}=0,0.4\right.$, and $1 \mathrm{eV}$ for Figs. 4(d), 4(e), and 4(f), respectively] and calculate the relative variation of the CLP at increasing values of $N$, normalizing with respect to the pressure with $N=1$. We see that for $\mu_{F}=$ $1 \mathrm{eV}$ the relative variation for $N=100$ goes up to $\simeq 80 \%$ [Fig. 4(f)]. It is worth stressing that in Fig. 4, by varying $N$ or $\mu_{F}$, the maximum variations are obtained at distances around $0.6 \mu \mathrm{m}$ and become negligible at a few microns, when the asymptotic universal regime $P_{\text {Lif }}$ is reached.

In order to have more insight on the origin of the large CLP modulation (Figs. 2 and 3) with respect to the temperature $T$, the Fermi level $\mu_{F}$, and the number of layers $N$, we first look, in Fig. 5, at the graphene conductivity $\sigma(i \xi)$ as a function of $T$ and $\mu_{F}$. After, in Fig. 6, we see how $\sigma(i \xi)$, jointly with the $\mathrm{SiO}_{2}$ permittivity $\varepsilon(i \xi)$ and the variation of $N$, affect the multilayer reflection coefficient $R_{p}^{(i)}\left(Q, i \xi_{n}, T\right)$.

The large thermal variation observed in Fig. 2 derives from strong thermal variations of $\sigma(i \xi)$ [see Fig. 5(b)], which directly affect the multilayer TM reflectivity $|R|^{2}$ at normal incidence $(Q=0)$ as shown in Fig. 6(a). In Figs. 5(b) and 5(c), we see the interplay between $T$ and $\mu_{F}$ encoded in Eq. (3), which implies that a larger relative thermal variation is obtained for $\mu_{F}=0 \mathrm{eV}$ (for larger doping, rapidly $\mu_{F} \gg k_{B} T \simeq 10^{-2} \mathrm{eV}$, implying no thermal conductivity effects). This explains the partial recovering of the TGA for $\mu_{F}=0 \mathrm{eV}$ in Figs. 2(a) and 2(b) and, on the other side, explains that the TMA recovered in Fig. 2(b) for $\mu_{F}=1 \mathrm{eV}$ is not due to thermal features of the graphene sheets. In Fig. 6(a), we see that the thermal variation of $\sigma(i \xi)$ affects the reflectivity mainly at frequencies smaller than $\simeq 10^{15} \mathrm{rad} / \mathrm{sec}$ [which are the dominating frequencies in the

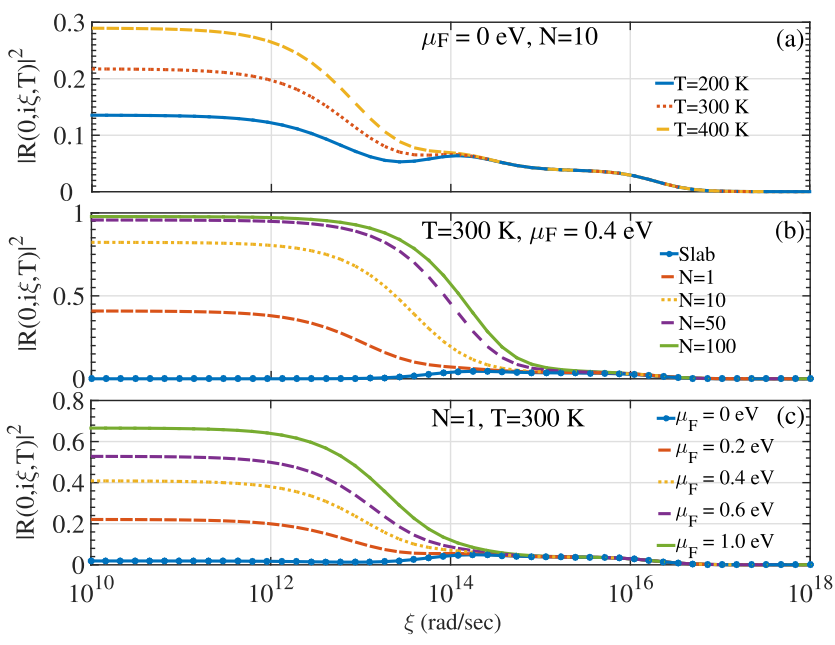

FIG. 6. TM reflectivity $|R|^{2}$ at normal incidence $(Q=0)$ at imaginary frequencies and for $L=1 \mu \mathrm{m}$. (a) $N=10$, $\mu_{F}=0 \mathrm{eV}$, and $T$ varies. (b) $T=300 \mathrm{~K}, \mu_{F}=0.4 \mathrm{eV}$, and $N$ varies. (c) $T=300 \mathrm{~K}, N=1$, and $\mu_{F}$ varies.

Matsubara sum (2)], while at larger frequencies the reflectivity is influenced only by the thermal-independent $\mathrm{SiO}_{2}$ dielectric permittivity $\varepsilon(i \xi)$ given in Fig. 5(a).

It is worth stressing that $|R(Q=0)|^{2}$ in Fig. 6 is useful to understand the behavior of the CLP in general, where several Matsubara terms $\xi_{n}$ contribute to the sum (2). This is not the case for the large separation limit $d \rightarrow \infty$, for which one should consider only the first Matsubara term $\xi_{0}=0 \mathrm{rad} / \mathrm{sec}$ and after perform the integration over $Q$. In that case, the reflectivities reduce to the metallic limits $\left|R_{\mathrm{TM}}\left(\xi_{0}\right)\right|^{2}=1$ and $\left|R_{\mathrm{TE}}\left(\xi_{0}\right)\right|^{2}=0$ for any $Q \neq 0$ (the $Q \rightarrow 0$ and $\omega \rightarrow 0$ limit ordering matters). In Fig. 2, the CLP for $N=1$ and $N=10$ at intermediate distances $d \simeq 1 \mu \mathrm{m}$ is not saturated by the single $\xi_{0}$ term (which would be enough for the suspended graphene configuration-dotted line) due to the mixed graphenedielectric configuration.

Let us now analyze the effect of varying both $\mu_{F}$ and $N$ on $|R|^{2}$ : We see in Fig. 6(b) that adding and increasing the number of graphene sheets strongly modifies the reflectivity in a large range of frequencies $\lesssim 10^{15} \mathrm{rad} / \mathrm{sec}$, approaching more and more an ideal metallic behavior $|R|^{2}=1$ (while $|R|^{2} \simeq 0$ at small frequencies for simple slabs). Analogous variations of $|R|^{2}$ are shown if, at fixed values of $N$, the Fermi level increases, as shown in Fig. 6(c). The reflectivity increases considerably by increasing $N$ and/or $\mu_{F}$, which confers to the graphene multilayer a tunable metallic behavior and explains the strong modulations of the CLP observed in Fig. 4.

Conclusions.-We analyze, in terms of the graphene conductivity and of the structure reflection coefficients, both individual and collective effects of changing the temperature, the Fermi level, and the number of graphene sheets on the CLP between graphene-dielectric multilayer structures. We exploit the fact that by changing $T, \mu$, and $N$ 
it is possible to modulate the graphene (semi)metallic features and, hence, the reflectivity of the structure. For these structures, we found that the CLP can strongly depend on the temperature, implying a dramatic change with respect to both single suspended graphene sheets (more difficult to realize) and dielectric slabs and allowing the measurement of thermal effects at small separations. Relevant similarities with normal 3D metals are found in some conditions. We also show that a consistent modulation of the CLP can be obtained by varying the number of graphene sheets in the structure or the Fermi level. This latter variation can be done by simply changing the electrostatic potential of the graphene sheets and allows for a fast in situ tuning of the interaction, which is of clear experimental interest. A natural direct extension of this study is to consider nonordered graphene-dielectric multilayer structures in order to further sculpt the CLP. These findings offer several opportunities for both experimental Casimir investigations and for more applicative studies in micro- and nanomechanical devices.

We acknowledge Florian Bigourdan and George Hanson for useful discussions.

*Corresponding author.

mauro.antezza@umontpellier.fr

[1] H. B. G. Casimir, Proc. K. Ned. Akad. Wet. 51, 793 (1948)

[2] I. E. Dzyaloshinskii, E. M. Lifshitz, and L. P. Pitaevskii, Adv. Phys. 10, 165 (1961).

[3] E. M. Lifshitz, Zh. Eksp. Teor. Fiz. 29, 94 (1955) [Sov. Phys. JETP 2, 73 (1956)].

[4] D. Dalvit et al., Casimir Physics, Lect. Notes Phys. (Springer-Verlag, Berlin, 2011).

[5] H. B. Chan, V. A. Aksyuk, R. N. Kleiman, D. J. Bishop, and F. Capasso, Phys. Rev. Lett. 87, 211801 (2001).

[6] M. Bostrom and B. E. Sernelius, Phys. Rev. Lett. 84, 4757 (2000).

[7] M. Antezza, L. P. Pitaevskii, and S. Stringari, Phys. Rev. A 70, 053619 (2004).

[8] D. M. Harber, J. M. Obrecht, J. M. McGuirk, and E. A. Cornell, Phys. Rev. A 72, 033610 (2005).

[9] M. Antezza, L. P. Pitaevskii, and S. Stringari, Phys. Rev. Lett. 95, 113202 (2005).

[10] J. M. Obrecht, R. J. Wild, M. Antezza, L. P. Pitaevskii, S. Stringari, and E. A. Cornell, Phys. Rev. Lett. 98, 063201 (2007).

[11] A. O. Sushkov, W. J. Kim, D. A. R. Dalvit, and S. K. Lamoreaux, Nat. Phys. 7, 230 (2011).

[12] H. B. Chan, Y. Bao, J. Zou, R. A. Cirelli, F. Klemens, W. M. Mansfield, and C.S. Pai, Phys. Rev. Lett. 101, 030401 (2008).

[13] A. Lambrecht and V. N. Marachevsky, Phys. Rev. Lett. 101, 160403 (2008).

[14] F. Chen, U. Mohideen, G. L. Klimchitskaya, and V. M. Mostepanenko, Phys. Rev. Lett. 88, 101801 (2002).
[15] F. Intravaia, S. Koev, I. W. Jung, A. Alec Talin, P. S. Davids, R. S. Decca, V. A. Aksyuk, D. A. R. Dalvit, and D. López, Nat. Commun. 4, 2515 (2013).

[16] J. Zou, Z. Marcet, A. W. Rodriguez, M. T. H. Reid, A. P. McCauley, I. I. Kravchenko, T. Lu, Y. Bao, S. G. Johnson, and H. B. Chan, Nat. Commun. 4, 1845 (2013).

[17] R. Guérout, J. Lussange, H. B. Chan, A. Lambrecht, and S. Reynaud, Phys. Rev. A 87, 052514 (2013).

[18] R. Messina, P. A. Maia Neto, B. Guizal, and M. Antezza, Phys. Rev. A 92, 062504 (2015).

[19] A. G. Grushin and A. Cortijo, Phys. Rev. Lett. 106, 020403 (2011).

[20] F. S. S. Rosa, D. A. R. Dalvit, and P. W. Milonni, Phys. Rev. Lett. 100, 183602 (2008).

[21] D. Iannuzzi, M. Lisanti, and F. Capasso, Proc. Natl. Acad. Sci. U.S.A. 101, 4019 (2004).

[22] L. M. Woods, D. A. R. Dalvit, A. Tkatchenko, P. RodriguezLopez, A. W. Rodriguez, and R. Podgornik, Rev. Mod. Phys. 88, 045003 (2016).

[23] A. H. Castro Neto, F. Guinea, N. M. R. Peres, K. S. Novoselov, and A. K. Geim, Rev. Mod. Phys. 81, 109 (2009).

[24] J. F. Dobson, A. White, and A. Rubio, Phys. Rev. Lett. 96, 073201 (2006).

[25] G. Gómez-Santos, Phys. Rev. B 80, 245424 (2009).

[26] D. Drosdoff and L. M. Woods, Phys. Rev. B 82, 155459 (2010).

[27] V. Svetovoy, Z. Moktadir, M. Elwenspoek, and H. Mizuta, Europhys. Lett. 96, 14006 (2011).

[28] G. L. Klimchitskaya, U. Mohideen, and V. M. Mostepanenko, Phys. Rev. B 89, 115419 (2014).

[29] M. Bordag and I. G. Pirozhenko, Phys. Rev. D 91, 085038 (2015).

[30] V. B. Bezerra, G. L. Klimchitskaya, V. M. Mostepanenko, and C. Romero, Phys. Rev. A 94, 042501 (2016).

[31] A. A. Banishev, H. Wen, J. Xu, R. K. Kawakami, G. L. Klimchitskaya, V. M. Mostepanenko, and U. Mohideen, Phys. Rev. B 87, 205433 (2013).

[32] B. Guizal and M. Antezza, Phys. Rev. B 93, 115427 (2016).

[33] See Supplemental Material at http://link.aps.org/ supplemental/10.1103/PhysRevLett.118.126101 for derivation of the reflection coefficients for the graphene-dielectric multilayer.

[34] W. J. Tropf and M. E. Thomas, in Handbook of Optical Constants of Solids, edited by E. Palik (Academic, New York, 1998), Vol. III.

[35] L. D. Landau and E. M. Lifshitz, Electrodynamics of Continuous Media (Pergamon, New York, 1963).

[36] L. A. Falkovsky, J. Phys. Conf. Ser. 129, 012004 (2008).

[37] L. A. Falkovsky and A. A. Varlamov, Eur. Phys. J. B 56, 281 (2007).

[38] F. H. L. Koppens, D. E. Chang, and F. J. García de Abajo, Nano Lett. 11, 3370 (2011), and Supplemental Material.

[39] S. A. Awan, A. Lombardo, A. Colli, G. Privitera, T. S. Kulmala, J. M. Kivioja, M. Koshino, and A. C. Ferrari, 2D Mater. 3, 015010 (2016).

[40] The typical expression for $\sigma_{\text {inter }}$ is that of Eq. (6) in Ref. [36]: It is well suited for real frequencies, while it is not valid if $\omega=i \xi_{n}$ and $\mu=0 \mathrm{eV}$. We hence use its modified version, 
coming from Eq. (1) of Ref. [36]. We neglect here the nonlocal effects which can be included by using a more general wave-vector-dependent anisotropic conductivity $\sigma_{i j}(\omega, k)$ [37]. These effects are negligible at the dominant optical frequencies (relevant at typical separations) and do not affect the recovery of the universal large distance law (given by the zero frequency contribution), making reasonable the approximation $\sigma_{i j}(\omega, k) \rightarrow \sigma(\omega, k=0)$ in the CLP integral.

[41] For gold, we used the Drude permittivity $\varepsilon(\omega)=1-\omega_{p}^{2} /$ $\left(\omega^{2}+i \omega \gamma\right)$, with $\hbar \omega_{p}=9 \mathrm{eV}$ and $\hbar \gamma=35 \mathrm{meV}$. 